The Egyptian Journal of Hospital Medicine (October 2019) Vol. 77 (3), Page 5173-5182

\title{
Assessment of Myocardial Viability After Acute ST- Elevated Myocardial Infarction Using Stress Speckle Tracking Echocardiography and Cardiac MRI
}

Ahmed Saleh *, Ali El-Amin**, Mohamed El-Baz**, Hany Negm*

*Cardiology unit, Research Institute of Ophthalmology, Ministry of Scientific Research and Technology,

$* *$ Cardiology department,

Al-Azhar University, Cairo, Egypt

* Corresponding author: A. Saleh, salehcardio@hotmail.com

\begin{abstract}
Background: Speckle-tracking echocardiography has emerged as a unique technique for accurately evaluating myocardial function by analyzing the motion of speckles identified. Speckle-tracking measured under stress may offer an opportunity to improve the detection of dynamic regional abnormalities and myocardial viability.

Objective: The aim of the current study was to evaluate stress speckle tracking to detect myocardial viability in comparison to cardiac MRI in post-STEMI patients.

Patients and methods: 74 patients were prospectively enrolled in 18-month's study. Dobutamine stress echocardiography was performed 4 days post-infarction accompanied with automated functional imaging analysis of left ventricle during rest and then during low dose stress. All patients underwent a follow up stress echocardiography at 3 months with speckle tracking analysis. Cardiac MRI took place concomitantly at 4 days post-infarction and 3 months.

Results: Investigating strain rate obtained with stress speckle tracking after revascularization predicted the extent of myocardial scar, determined by contrast-enhanced magnetic resonance imaging. A good correlation was found between the global strain and total infarct size $(\mathrm{R} 0.75, \mathrm{p}<0.001)$. Furthermore, a clear inverse relationship was found between the segmental strain and the transmural extent of infarction in each segment. Meanwhile it provided $81.82 \%$ sensitivity and $82.6 \%$ specificity to detect transmural from non-transmural infarction at a cut-off value of -10.15 .

Conclusion: Strain rate obtained from speckle tracking during stress is a novel method of detecting myocardial viability after STEMI. Moreover, it carries a promising role in post-myocardial infarction risk stratification with a reasonable prediction of reversible cardiac-related hospital re-admission.
\end{abstract}

Keywords: STEMI, stress echocardiography, speckle tracking, Cardio-MRI.

\section{INTRODUCTION}

Stress echocardiography has emerged as an important modality for the assessment of patients with coronary artery disease (CAD) and left ventricular (LV) systolic dysfunction. Multicenter studies have shown worse outcome when viable myocardium was identified by stress echocardiography but the patient was not revascularized ${ }^{(1)}$.

Most stress echocardiography protocols are centered on the detection of contractile reserve and have used inotropic stimulation with dobutamine ${ }^{(1)}$.

Speckle-tracking echocardiography (STE) has recently emerged as a quantitative ultrasound technique for accurately evaluating myocardial function by analyzing the motion of speckles identified on routine 2-dimensional sonograms ${ }^{(2,3,4)}$.

Thus a new based on Speckle tracking imaging technique, automated function imaging (AFI) allowing a faster assessment of regional longitudinal strain of the left ventricle and clear results presentation as a polar map has recently gained wide interest and has been tested in various clinical settings although its applicability for DSE (Dobutamine stress echocardiography) is still poorly documented ${ }^{(5)}$.

In the acute setting, the extent of late gadolinium enhancement in cardiac MRI is related to the magnitude of cardiac enzyme release and the functional outcome after recovery. Late gadolinium enhancement CMR reveals a permanent record of MI (both acute and chronic) and is proving very useful clinically for the diagnosis of MI in cases of doubt ${ }^{(6,7)}$.

Further work has shown that CMR can differentiate between the densely bright infarct core and the adjacent peri-infarct zone, which appears with lower signal intensity because of the admixture of infarct and viable tissue. The greater the extent of the peri-infarct zone, the greater the likelihood of future cardiac death and events ${ }^{(8)}$.

The aim of the current study was to evaluate the stress speckle tracking in detection of functional recovery and viability of global and regional 
myocardial function following successful revascularization in STEMI patients in comparison to cardiac MRI.

\section{PATIENTS AND METHODS}

This prospective study included a total of 74 myocardial infarction patients diagnosed with ST elevation based on their ECG, laboratory findings and symptoms at admission, attending at Cardiology unit, Research Institute of Ophthalmology, Ministry of Scientific Research and Technology.

\section{Ethical approval:}

Written informed consent from all the subjects were obtained. This study was conducted between Mai 2014 until December 2015. The study was approved by the Ethics Board of Al-Azhar University.

Dobutamine stress echocardiography was performed 4 days post-infarction accompanied with automated functional imaging analysis of left ventricle during rest and then during low dose stress. All patients underwent a follow up stress echocardiography at 3 months with speckle tracking analysis. Cardiac MRI took place concomitantly at 4 days post-infarction and 3 months.

Exclusion Criteria: Patients with very poor acoustic window due to marked obesity or Chronic Obstructive Pulmonary Disease (COPD) and patients with either ventricular or supraventricular arrhythmia with risk to perform a stress study were excluded. Meanwhile Patients who are contraindicated to perform a cardiac MRI test (pacemaker carriers, cochlear implants and patients with diagnosed claustrophobia) were likewise ruled out.

\section{Echocardiography}

Examinations were performed with a digital ultrasonic device system (Vivid 9, GE Vingmed Ultrasound, Horten, Norway). The patients were examined 96 hours after revascularization using M4S probe in left lateral position. In harmonic mode 2.0/4.3 MHz with maximal frame per second (FPS) count available at necessary sector width. Range of FPS was from 64 to 112 with mean value 83 .

Conventional echocardiography study was done including 2D based M-mode measures of cardiac chambers and Ejection Fraction (EF\%), pulsed and continuous wave Doppler studies, Color Doppler study, calculation of ejection fraction by Simpson`s method and analysis of wall motion abnormalities. Regional wall motion abnormalities were analyzed using wall motion score.

\section{Dobutamine stress Echocardiography}

The patients underwent low dose dobutamine stress echocardiography using a digital ultrasonic device system (Vivid 9, GE Vingmed Ultrasound, Horten, Norway). The patients were examined 96 hours after revascularization using $\mathrm{M} 4 \mathrm{~S}$ probe in left lateral position. In harmonic mode $2.0 / 4.3 \mathrm{MHz}$ with maximal frame per second (FPS) count available at necessary sector width.

4 days post-STEMI as well as a full dobutamine stress testing follow up study at 3 months.

- Patients were prepared for standard stress testing.

- Intravenous access was obtained.

- Digital images were acquired at baseline (these loops are displayed and used as reference throughout the infusion).

- Continuous electrocardiogram and blood pressure monitoring were established.

- Dobutamine infusion began at a dose of 10 $\mu \mathrm{g} / \mathrm{kg} / \mathrm{min}$.

- The infusion rate is increased every 3 minutes to doses of 20 at low dose and 30, and $40 \mu \mathrm{g} / \mathrm{kg} / \mathrm{min}$. for complete stress study.

- Peak images were acquired before termination of the infusion.

- Post-stress images were recorded after return to baseline.

- The patient was monitored until he/she returns to baseline status.

Whereas atropine was added in $0.5 \mathrm{mg}$ fractional doses after the second stage of infusion up to the total dose of $2 \mathrm{mg}$ till the target heart rate was achieved. The infusion of dobutamine was stopped when age-adjusted HR limit was reached or when patients experiences criteria for terminating (angina pectoris or dyspnea, arrythmias and other serious conditions).

\section{Strain rate obtained by Speckle Tracking study}

Left ventricle (LV) was recorded in 2D cine-mode in 3 views: - apical 4 chambers, apical 2 chambers and apical 3 chambers. Then LV was studied by offline analysis through the external work station. Three points were selected in each view ( 2 points at the base and 1 point at the apex) then the program automatically tracked the endocardial border of LV in each view. Regional and global strain of LV were calculated automatically and expressed in Bull's eye form based on 17 myocardial segmentation system that showed average global strain and peak systolic strain of each myocardial segment giving it a specific color according to value of peak systolic strain. 


\section{MR protocol}

CMR was performed using a 3T scanner (MRI Verio, Siemens, Erlangen, Germany). Steady-state free precession cine images were used to quantify LV function (slice thickness of $6 \mathrm{~mm}$ ), and LV short-axis images were acquired from apex to base to cover entire LV volumes. Repeated breath-holds were required in order to create adequate images. The temporal resolution was 25-30 frames per RR interval. LV mass was measured with commercially available software (CVI42, Circle Cardiovascular Imaging Inc., Calgary, Canada) by a single experienced observer (SCMR Level III) unaware of echocardiographic data. LGE (late gadolinium enhancement) images were acquired 10 minutes after the intravenous administration of Gadoterat-Meglumine $\quad(0.2 \mathrm{mmol} / \mathrm{kg}$ intravenous injection, Dotarem; Guerbet), and followed by a flush of $20 \mathrm{~mL}$ of saline at the same rate. LGE images from long-axial, short-axial, and 4-chamber views were acquired using the inversion recovery segmented spoiled-gradient echo and phase-sensitive inversion recovery methods. LGE images were evaluated using a 17-segment model, as suggested by the American Heart Association criteria.

\section{Data analysis}

Imaging data were analyzed with a commercially available postprocessing workstation (CVI42, Circle Cardiovascular Imaging Inc., Calgary, Canada). Endocardial and epicardial contours were traced manually on short-axis cine MR images of the LV at end diastole and end systole to assess end-diastolic and endsystolic volumes, LV ejection fraction, and LV mass. In order to assess the presence or absence of LGE and its extent, all short-axis slices from base to apex were inspected visually to identify areas of normal (completely nulled) myocardium. Contrast-enhanced images were analyzed by two experienced observers unaware of other CMR, echocardiographic, and clinical data. Any discrepancy in analysis between the 2 readers was resolved by a senior observer (SCMR Level III.). Additionally, LGE in each of the 17 segments was graded into subendocardial scar $(<75 \%$ of the wall diameter) or transmural scar ( $>75 \%$ of wall diameter).

\section{Statistical analysis}

Statistical analysis was performed using MedCalc V.12.1.4. (Frank Schoonjans Belgium). Receiveroperating characteristic (ROC) curves were constructed, and areas under curves were measured to determine cutoff values with maximum sensitivity and specificity. A p value $<0.05$ was considered statistically significant. We performed a multivariate regression analysis to find the best method for estimating final infarct size utilizing cardiac MRI as the reference method.

\section{RESULTS}

\section{Demographics}

Women accounted for $49 \%$ of our study cohort whereas Men were $51 \%$ of the patients. Forty-six patients were hypertensive while thirty-two were already diagnosed with diabetes before admission with STEMI. Forty patients of the studied group were smokers (54\%) while thirty-five showed either elevated lipid profile or were taking lipid-lowering drugs. Nine patients had experienced a previous acute coronary syndrome in their medical history while twelve of them had previous coronary interventions (PCI) with stent implantation. (Table 1).

(Table 1): Demographics of patients who were enrolled in the study. ACS (Acute coronary syndrome). PCI (percutaneous coronary intervention).

\begin{tabular}{|l|l|l|}
\hline $\begin{array}{l}\text { Gender } \\
\text { Male }\end{array}$ & Number & Percentage \\
\hline $\begin{array}{l}\text { Female } \\
\text { Hypertension }\end{array}$ & 38 & $51 \%$ \\
$\begin{array}{l}\text { Non- } \\
\text { hypertensive }\end{array}$ & 28 & $49 \%$ \\
$\begin{array}{l}\text { Hypertensive } \\
\text { Diabetes }\end{array}$ & 46 & $37.9 \%$ \\
mellitus & 42 & $62.1 \%$ \\
\hline $\begin{array}{l}\text { Non-diabetics } \\
\text { Diabetics }\end{array}$ & 32 & $56.8 \%$ \\
\hline $\begin{array}{l}\text { Smoking } \\
\text { Non-smoker }\end{array}$ & 34 & $43.2 \%$ \\
Smoker & 40 & \\
\hline Hyperlipide & & $46 \%$ \\
mia & 39 & $54 \%$ \\
No & 35 & $52.7 \%$ \\
Yes & & $47.3 \%$ \\
\hline $\begin{array}{l}\text { Previous ACS } \\
\text { No }\end{array}$ & 65 & \\
Yes & 9 & $93.3 \%$ \\
Previous PCI & & $6.7 \%$ \\
No & 63 & $91.2 \%$ \\
Yes & 11 & $8.8 \%$ \\
\hline
\end{tabular}


The mean age in our study group was 64 years old \pm 9.7 and mean Body mass index was $25 \mathrm{~kg} / \mathrm{m}^{2} \pm 3.7$. Troponin levels at 4 days post-infarction were $0.65 \mathrm{ng} / \mathrm{ml} \pm 0.8$ while at the control exam at 3 months were $0.24 \mathrm{ng} / \mathrm{ml} \pm$ 0.4. Pro-BNP levels at 4 days were $696.0 \mathrm{pg} / \mathrm{ml} \pm 404$ and at 3 months showed an improvement to a level of 5470 $\mathrm{pg} / \mathrm{ml} \pm 281$. (Table 2$)$

(Table 2) Mean and standard deviation of Age, Body mass index (BMI), Ejection fraction (EF), Global and regional strain at rest and under stress (SR-Rest, SR-Stress), Troponin levels and Pro-Brain Natriuretic Peptide at 4 days and 3 months.

\begin{tabular}{|c|c|c|c|c|c|}
\hline & $\begin{array}{l}\text { Minimum } \\
\text { / } \\
\text { Maximum }\end{array}$ & Mean & $\begin{array}{l}\text { Std. } \\
\text { Deviatio } \\
\text { n }\end{array}$ & $\begin{array}{l}\text { Student } \mathrm{t} \\
\text { test }\end{array}$ & $\begin{array}{l}\text { P value } \\
\text { Significanc } \\
\text { e }\end{array}$ \\
\hline Age (years) & $39-84$ & 64.39 & 9.781 & 46,08 & $<0,0001$ \\
\hline BMI $\left(\mathrm{kg} / \mathbf{m}^{2}\right)$ & $19-33$ & 25.41 & 3.774 & 47,12 & $<0,0001$ \\
\hline EF (4d) & $27-63$ & 47.41 & 8.715 & 38,07 & $<0,0001$ \\
\hline EF (3m) & $25-66$ & 49.67 & 8.680 & 40,05 & $<0,0001$ \\
\hline Global SR-Rest (4d) & $-7.5--18.3$ & -14.147 & 2.8157 & 35,16 & $<0,0001$ \\
\hline Global SR-Stress (4d) & $-6.9--22.5$ & -16.292 & 3.7278 & 30,59 & $<0,0001$ \\
\hline Global SR-Rest (3m) & $-7.1--19.9$ & -15.745 & 3.3786 & 32,62 & $<0,0001$ \\
\hline Global SR-Stress (3m) & $-6.5--22.8$ & -17.245 & 4.1164 & 29,32 & $<0,0001$ \\
\hline Regional SR-Rest (4d) & $7--13$ & -3.53 & 4.491 & 5,5 & $<0,0001$ \\
\hline Regional SR-Stress (4d) & $11--18$ & -8.27 & 5.674 & 10,19 & $<0,0001$ \\
\hline Regional SR-Rest (3 m ) & 4- - -16 & -7.65 & 4.608 & 11,62 & $<0,0001$ \\
\hline Regional SR-Stress (3 m) & $10--23$ & -13.86 & 6.272 & 15,46 & $<0,0001$ \\
\hline Troponin-4d & $0.01-4.04$ & 0.6544 & 0.82580 & 5,54 & $<0,0001$ \\
\hline Troponin-3 m & $0.00-1.90$ & 0.2458 & 0.40400 & 4,25 & $<0,0001$ \\
\hline Pro-BNP-4d & $73-12,377$ & 696.0 & 404 & 3,92 & $<0,0001$ \\
\hline Pro-BNP- $3 \mathrm{~m}$ & $54-14,512$ & 547.0 & 281 & 3,76 & $<0,0001$ \\
\hline
\end{tabular}

Gender difference was not significant in both global and regional, resting and stress strain obtained with speckle tracking neither at 4 days nor at 3 months, $p$ value $>0.5$. Diabetes mellitus (DM) was highly significant in affecting global strain rate at rest and at stress on 4 days, $p$ value 0.017 and 0.001 respectively. At the follow up, DM was not significant at rest or at stress. Both smoking and Hyperlipidemia have not shown any significance in relation to global and regional, resting and stress strain obtained with speckle tracking. The patients with previous stenting had significantly lower global and regional strain rate at stress at both 4 days and 3 months in comparison to patients with no history of PCI, p value 0.02 and 0.12 respectively. The significance of such values was determined on the basis of improvement or worsening the strain rate values in resting and stress studies at 4 days and at 3 months of follow up.

\section{Laboratory Results Analysis}

Troponin levels at 3 months showed a moderate correlation with global stress strain obtained with speckle tracking at 3 months with $r$ value of 0.43 and $p$ value $<0.001$ (Figure 1). ProBNP exhibited a moderate correlation with with global stress strain obtained with speckle tracking at 3 months with $r$ value of 0.41 and $p$ value of 0.003 . 


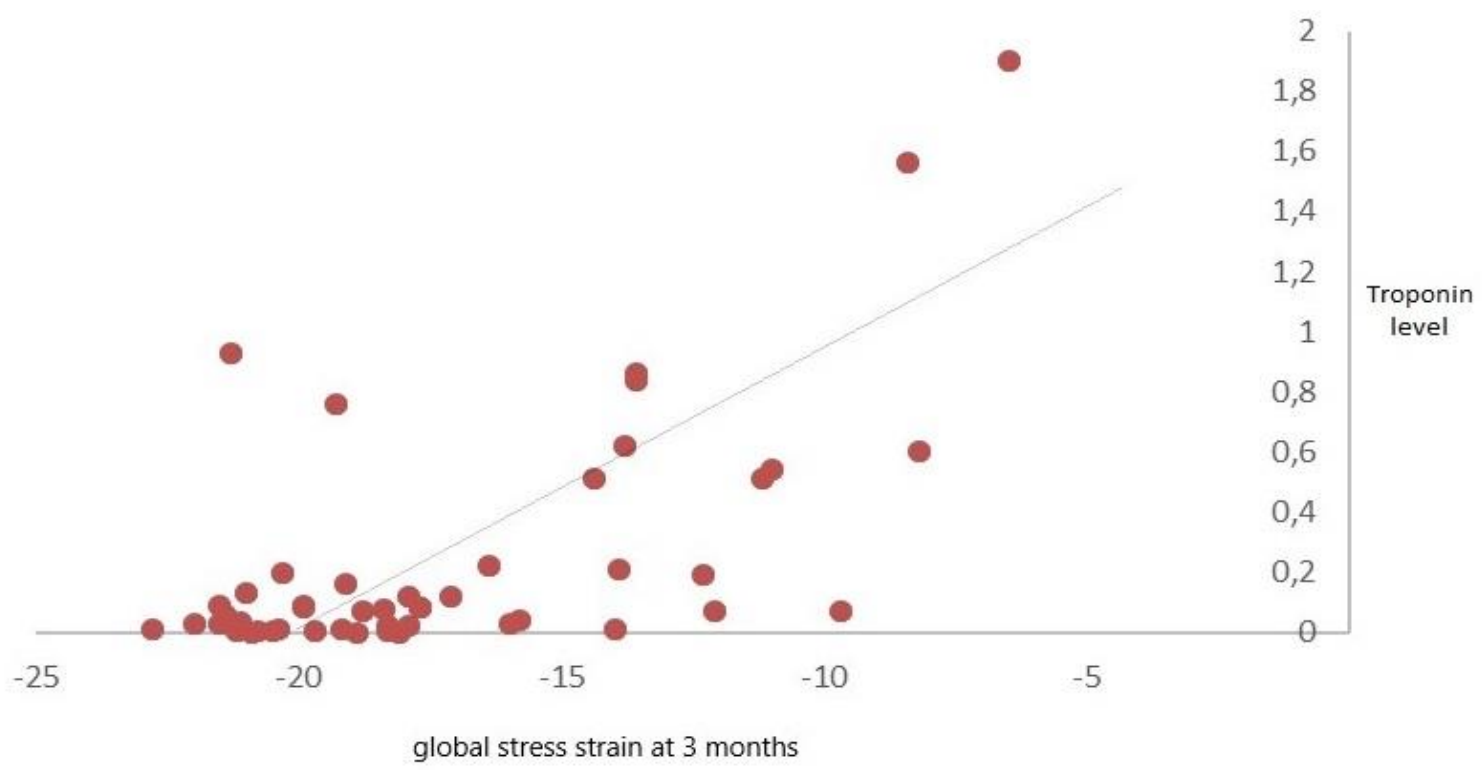

Figure (1): Correlation between Troponin levels and global stress strain at 3 months.

\section{Imaging Tools Analysis:}

The total enhancement mass obtained by MRI showed a mean value of $29 \pm 11$ in patients with transmural scar while it showed a mean value of $10 \pm 8$ in patients with subendocardial scar. No statistical relationship was detected between acute myocardial volume, which was extracted from MRI, and stress strain parameters.

A statistical agreement was conducted to detect the most appropriate method in determining post-infarction ischemia at 4 days in relation to cardiac MRI at the different level of myocardial walls. Dobutamine stress echocardiography revealed a kappa value of 0.72 with a p value of 0.04 while global strain rate at rest showed a kappa value of 0.79 with a $\mathrm{p}$ value of 0.006 . Global strain rate under stress had a kappa value of 0.89 with a $\mathrm{p}$ value of 0.0001 (Figure 2).

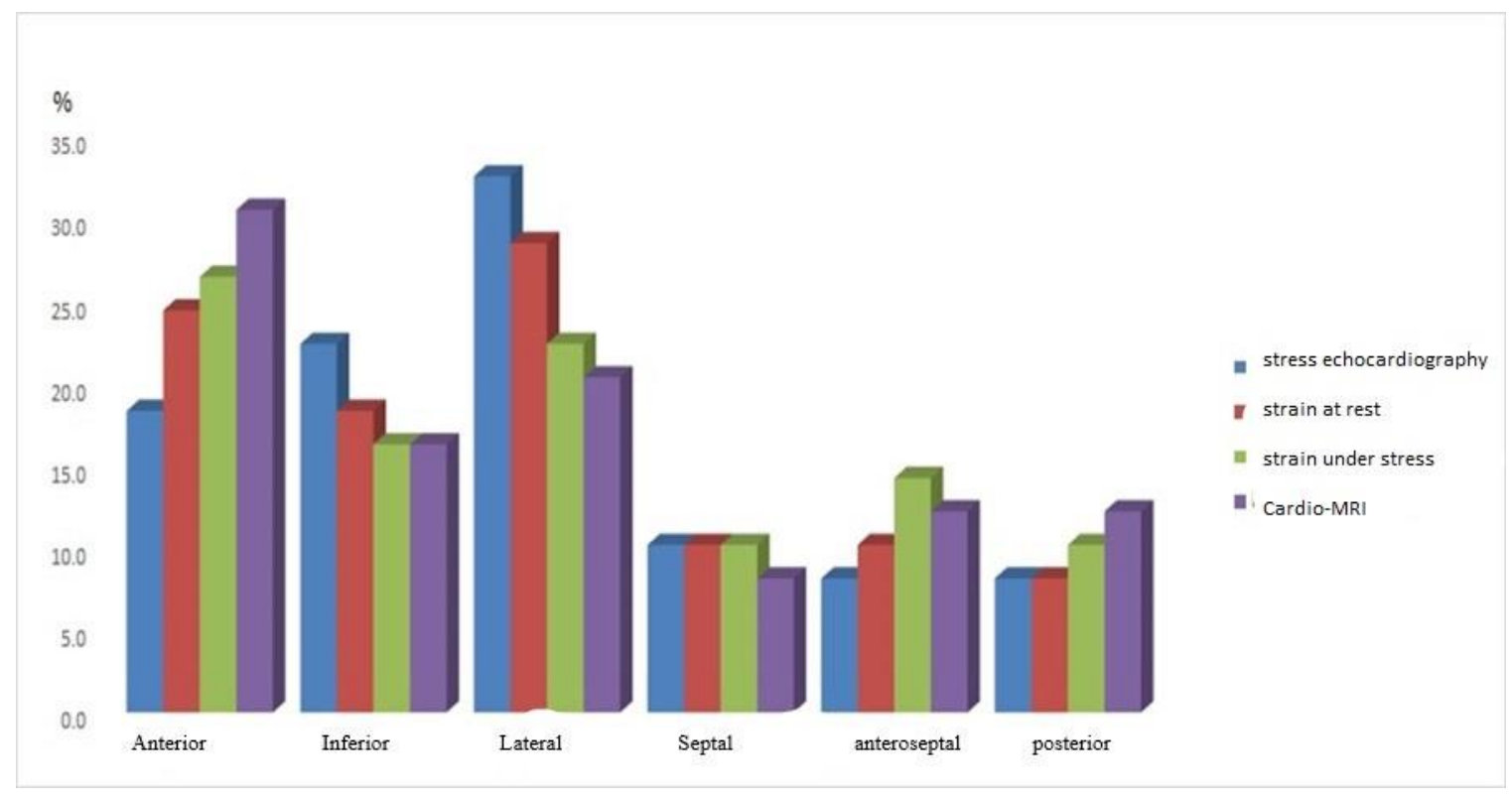

Figure (2): Agreement analysis at 4 days comparing different echocardiographic methods to cardiac MRI in detecting post-MI ischemia. 
Moreover, the same analysis was performed to distinguish the different echocardiographic data obtained through dobutamine stress, resting strain rate and stress strain rate at 4 days in correlation to the ischemic territory in coronary angiography. In this analysis an agreement was found between wall motion analysis revealed by dobutamine stress echocardiography with kappa value of 0.25 and $p$ value of 0.1 . Using resting strain revealed an agreement with a kappa value of 0.32 and $p$ value of 0.04 . Obtaining strain rate at stress raised the agreement to a kappa value of 0.46 with a $\mathrm{p}$ value of 0.001 (Figure 3 ).

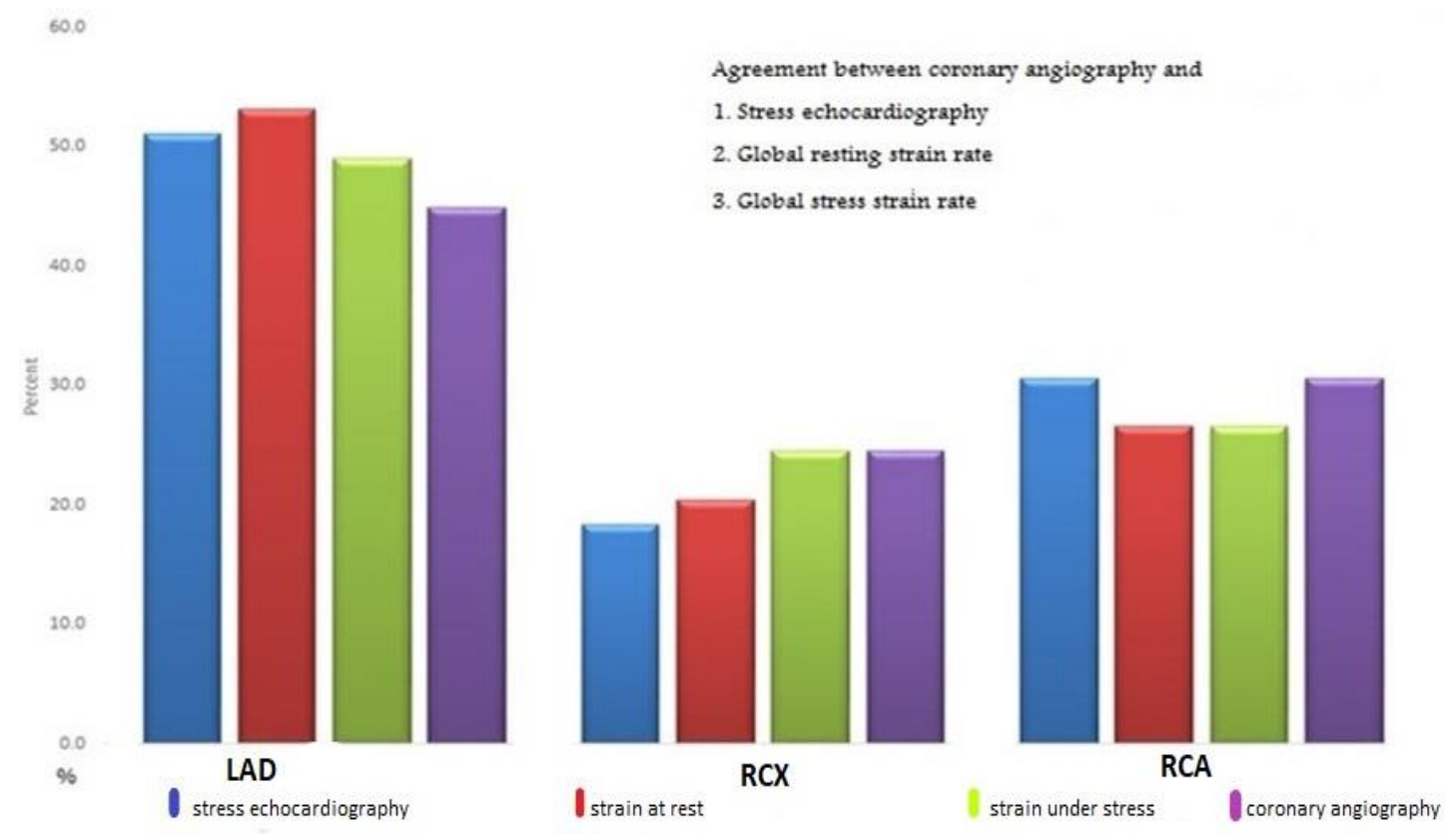

Figure (3): Agreement analysis at 4 days comparing different echocardiographic methods to coronary angiography findings in detecting post-MI ischemia.

Global stress strain rate obtained from speckle tracking showed different levels in relation to the MI extent at 3 months. A sub-endocardial MI ( $<75 \%$ extension of left ventricular wall as measured by cardiac MRI) had a mean value of $-18,9 \pm 4.8$ while a transmural infarction ( $>75 \%$ involvement of left ventricular wall) indicated a mean value of $-4.9 \pm 4.4$ (Figure 4$)$.

subendocardial MI

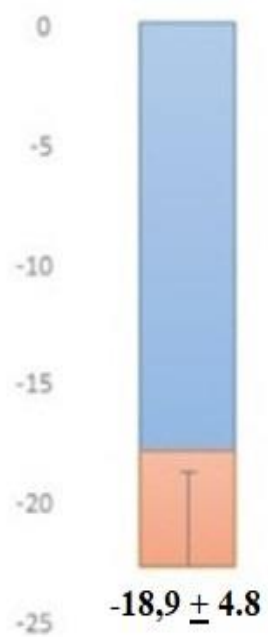

Transmural MI

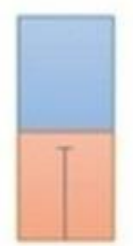

$-4.9 \pm 4.4$

mean values of strain under stress

standard deviation of strain under stress

Figure (4): Mean values and standard deviation of global stress strain obtained by speckle tracking as differentiated by extent of infarction in cardiac MRI. 
Regional stress strain rate at 3 months also was able to discriminate various forms of myocardial infarction extension through left ventricular wall with a mean value of $-19.5+3.9$ in sub-endocardial infarction. Furthermore, a mean value of $-1.9 \pm 2.5$ was demonstrated in patients with transmural MI.

We conducted a Receiver-operating characteristics (ROC analysis) in order to detect the sensitivity and specificity of various diagnostic modus obtained from echocardiography (normal stress echocardiography, resting strain stress obtained by speckle tracking and speckle tracking stress strain stress) in relation to cardiac MRI viability.

It revealed a $60 \%$ sensitivity and $74.29 \%$ specificity while applying wall motion abnormalities detected by dobutamine stress echocardiography (AUC, Area under the curve 0,677). Global resting strain rate was $74.09 \%$ sensitive and $60 \%$ specific in detecting myocardial viability in relation to cardiac MRI (AUC 0.764 ) at a cut-off value of -10.4 in differentiating transmural from subendocardial infarction. Obtaining strain rate under stress by speckle tracking provided an $81.82 \%$ sensitivity and $82.6 \%$ specificity to detect transmural from subendocardial infarction at a cut-off value of -10.85 .

We performed a multivariate regression analysis to find the best method for detecting myocardial viability at 3 months, using Cardiac MRI as the reference method. That disclosed a regression coefficient of 0.182 with significance level of $p$ value 0.0022 as of using dobutamine stress in detecting viability. Utilization of regional strain rate showed a regression coefficient of 0.168 with a $p$ value of 0.0034 . A regression of 0.501 with a significance level of $<0.0001$ was raised by using global strain rate at rest.

Regional strain rate values under stress divulged a highly significant regression with a coefficient of 0.776 and a $\mathrm{p}$ value $<0.0001$. While the highest significance was obtained by global stress strain rate with a regression coefficient of 0.8056 and a significance level of $<0.0001$.

Two years follow up of the patients revealed 7 cases of hospital re-admission due to cardiology-related symptoms (5 patients with recurrent chest pain, one patient due to dyspnea on mild exertion NYHA III and one patient with cardiac syncope). Global stress strain rate obtained from speckle tracking showed $80 \%$ sensitivity and $77.5 \%$ specificity at a cut-off value of 9.1 to predict hospital re-admission.

From those seven patients, three were diagnosed with acute coronary syndrome depending on the laboratory findings and ECG changes (one patient with unstable angina pectoris and one patient with NSTEMI). A cut-off value of -8.4 had shown a $69.23 \%$ sensitivity and $73.5 \%$ specificity to predict the recurrence of acute coronary syndrome.

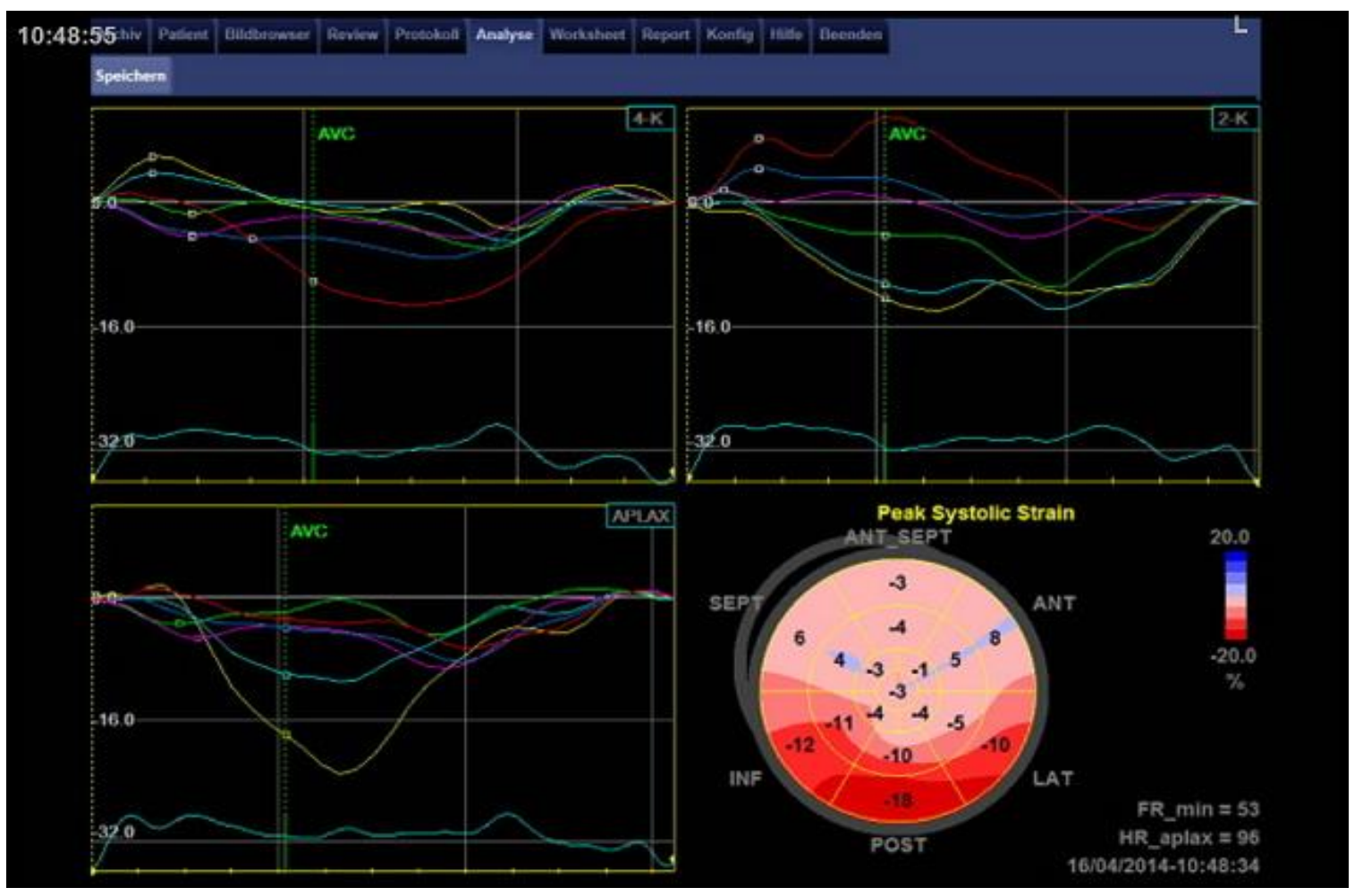




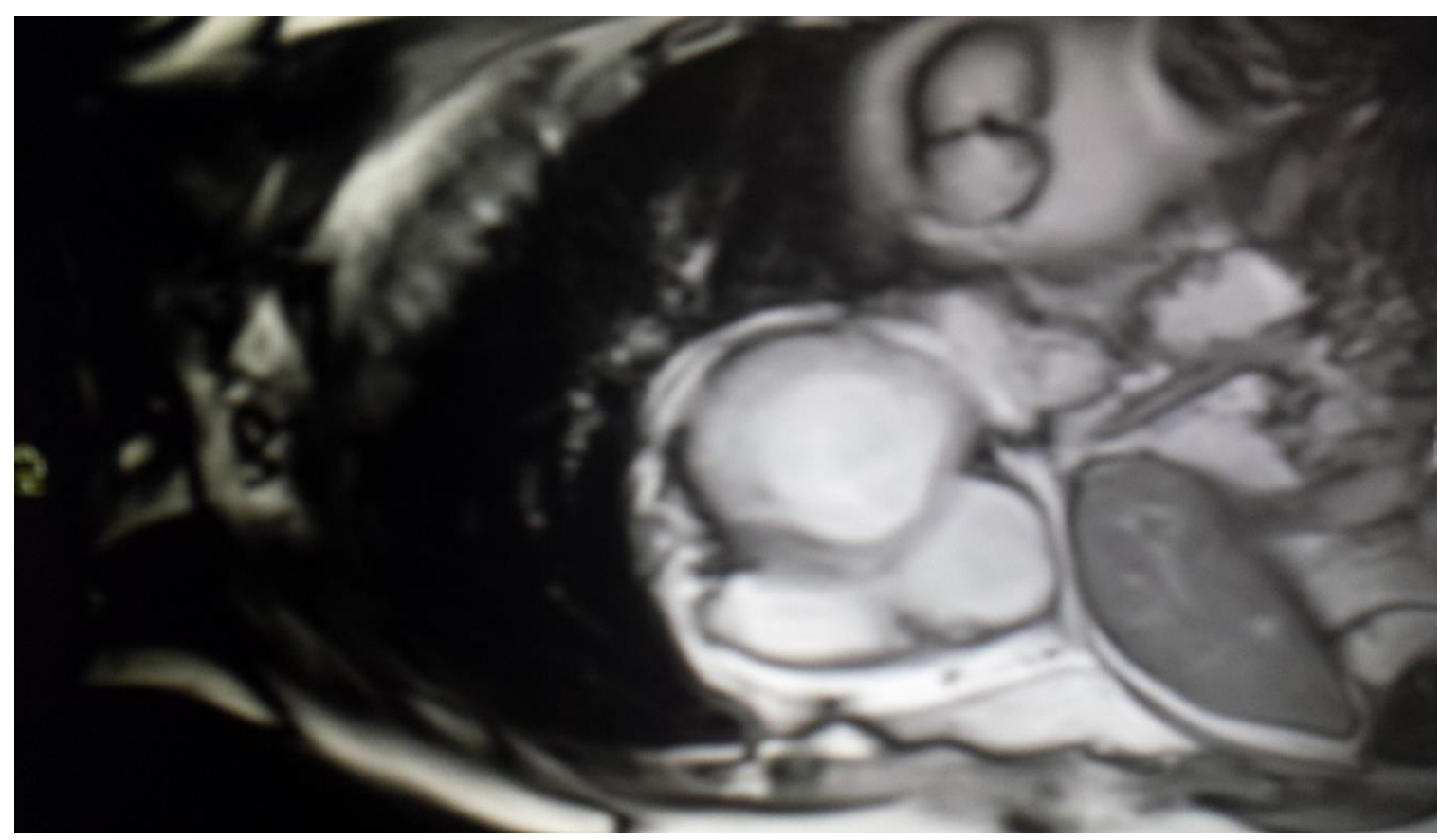

Figure (5): Stress speckle tracking against cardiac MRI at 3 months interval revealing a transmural involvement of myocardium (Anterior).

\section{DISCUSSION}

The need to provide a feasible, rapid and accurate tool to detect viability post-myocardial infarction is crucial. Thus, we studied the application of strain obtained during rest and stress in comparison to cardiac MRI as gold standard method.

Our laboratory findings, Troponin and Pro-BNP, showed a significant correlation with strain at rest and under stress. However, the correlation was higher in case of strain under stress revealing that the underlying hibernated myocardium reacts to stress. Meanwhile, the differentiation of stunned and hibernated myocardium is suboptimal at resting conditions as shown by strain results at rest.

The utility of strain obtained by speckle tracking was more accurate and presented higher sensitivity and specificity in comparison to regular stress echocardiography. The fact that strain values represents the smallest change in elongation and shortening of cardiac speckles adds a great value to its accuracy in comparison to traditional echocardiography. The relevant correlations and agreement which were revealed to the gold standard methods, cardiac-MRI in viability and coronary angiography in stenotic coronary vessels, confirm it as a promising tool which can replace traditional stress echocardiography.

A Norwegian experimental study, which took place in 2010 tried to simulate a clinical stress test applied in coronary artery disease with a constant coronary stenosis of about $80 \%{ }^{(9)}$. The study demonstrated that the spatial sensitivity of the speckle tracking highresolution ultrasound allows a focused assessment of specific layers of the myocardium and a graded assessment of parts of the myocardial wall with a different sensitivity to reduced coronary reserve. Thus, when applying an inotropic challenge to the hypoperfused ventricular wall, the midmyocardial layers in longitudinal and circumferential directions demonstrated the highest sensitivity by increasing the deformation measured by ET strain. This heterogeneous response was also evident by the lack of response of subendocardial and midendocardial radial ET strain. This observation is compatible with previous studies ${ }^{(10)}$, as submidendocardial radial ET strain might need cross-fiber activation of all layers and therefore might not be significantly activated when not all myocardial layers normalize their contractility ${ }^{(11)}$.

These findings were reproducible on the level of longitudinal strain at stress in our study showing the importance of applying stress to allow proper assessment of hypoperfused myocardium through inotropic challenge.

Hong Ran et al. ${ }^{(12)}$, evaluated in 2012 whether myocardial strain under adenosine stress calculated from two-dimensional echocardiography by automatic frame-by-frame tracking of natural acoustic markers 
enables objective description of myocardial viability in clinic. Two-dimensional echocardiography and twodimensional speckle tracking imaging (2D STI) at rest were performed first and once again after adenosine was infused at $140 \mathrm{ug} / \mathrm{kg} / \mathrm{min}$ over a period of 6 minutes in 36 stable patients with previous myocardial infarction. Radionuclide myocardial perfusion/metabolic imaging served in his study as the "gold standard" to define myocardial viability.

In the nonviable group, strain values showed no significant changes during adenosine administration. After adenosine administration, RS peak-sys and LS peak-sys in viable group increased significantly compared with nonviable group. Obtained strain data were highly reproducible and affected in small intraobserver and interobserver variabilities. A change of radial strain more than $9.5 \%$ has a sensitivity of $83.9 \%$ and a specificity of $81.4 \%$ for viable whereas a change of longitudinal strain more than $14.6 \%$ allowed a sensitivity of $86.7 \%$ and a specificity of $90.2 \%{ }^{(13)}$. In our study, cardiac MRI was manifested as gold standard to detect viability instead of radionuclide myocardial perfusion. Our strain data were reproducible, didn't present inter- or intraobserver variations and carried comparable high sensitivity in detection of viable myocardium. Moreover, we stated a cut off value to differentiate a subendocardial scar from an endocardial one.

In 2005, Zhang et al studied longitudinal strain to determine the value of regional deformation when compared to cardiac MRI. SRs and SRe were significantly lower in subendocardial infarction compared with normal myocardium. Moreover, they established a direct relationship between SRI, transmurality, and infarct size. Linear regression analysis showed that both SRs and Sm of the infarct area had a significant correlation with the infarct size. SRs of the transmural infarcted segments were significantly decreased when compared with nontransmural, subendocardial MI, and normal segments; SRs and SRe were also significantly reduced in subendocardial infarction compared with normal subjects ${ }^{(14)}$.

We used the global definition of myocardial scars dividing them into subendocardial and myocardial involvement. Strain values at rest and under stress were significantly lower in case of transmural affection of myocardium in comparison to a subendocardial myocardial scar.

A polish study assessed correlations between STE and AFI derived peak systolic longitudinal strain values for global and regional parameters, feasibility, time of analysis and interobserver agreement. The novel, simplified AFI technique offered similar feasibility, lower coefficient of variance both during rest and peak stress stage significantly (about two times) shorter time needed for analysis ${ }^{(15)}$.

Investigating strain Doppler echocardiography performed immediately after revascularization by percutaneous coronary intervention predicted the extent of myocardial scar, determined by contrast-enhanced magnetic resonance imaging. A good correlation was found between the global strain and total infarct size $(\mathrm{R}$ $0.77, \mathrm{p}<0.00001)$. A multi-variate regression analysis showed that global peak strain and serum glutamic oxaloacetic transaminase correlated with the infarct size measured by MRI $(\mathrm{p}<0.0001$ and $\mathrm{p}<0.001$, respectively). Furthermore, a clear inverse relationship was found between the segmental strain and the transmural extent of infarction in each segment ${ }^{(16)}$.

Likewise, a recently published Dutch study revealed that stress speckle tracking represents a promising new technique to detect significant angiographic CAD at follow-up ${ }^{(17)}$. Furthermore, stress speckle had proven a positive role in detection of sub-clinical LV hypertrophy in hypertensive patients ${ }^{(18)}$.

Our obtained data revealed a significant correlation of strain values at rest and under stress in relation to the stenotic coronary artery. The Highest agreement were shown using strain under low stress to detect acute ischemia post myocardial infarction. The numerical values of obtained strain were able to overcome the traditional assessment of wall motion abnormalities under stress in echocardiography which is based on the individual evaluation of cine-loops, which varies according to the examiner and his experience in the field.

\section{CONCLUSION}

Strain rate obtained from speckle tracking during stress is a novel method of detecting myocardial viability after STEMI and provide dependable results in comparison to strain rate obtained at rest.

It is safe, fast and reproducible and elucidates the Inter-Intraobserver variability of normal stress echocardiography. Meanwhile stress speckle tracking is highly sensitive and specific in correlation to the gold standard method (cardiac MRI).

Moreover, it carries a promising role in postmyocardial infarction risk stratification with a reasonable prediction of reversible coronary events or hospital re-admission. However, more data is still needed to confirm this emerging role. 


\section{REFERENCES}

1. Afridi I, Grayburn P, Panza J et al. (1998): Myocardial viability during dobutamine echocardiography predicts survival in patients with coronary artery disease and severe left ventricular systolic dysfunction. J Am Coll Cardiol.,32:921-6.

2. Götte MJ, Germans T, Rüssel K et al.(2006): Myocardial strain and torsion quantified by cardiovascular magnetic resonance tissue tagging studies in nor-mal and impaired left ventricular function. J Am Coll Cardiol., 48:20022011.

3. Elmayergi NH, Goodman JM, Lee LS, Sasson $\mathrm{Z}$ et al.(2013): Are measures of left ventricular systolic performance during low dose dobutamine stress echocardiograms repeatable over time? Int J Cardiovasc Imaging, 29:1281-1286.

4. Moon JC, De Arenaza DP, Elkington AG et al. (2004): The pathologic basis of Q-wave and non-Q-wave myocardial infarction: A cardiovascular magnetic resonance study. J Am Coll Cardiol., 44:554.

5. Wagner A, Mahrholdt H, Holly TA et al.(2003): Contrast-enhanced MRI and routine single photon emission computed tomography (SPECT) perfusion imaging for detection of subendocardial myocardial infarcts: An imaging study. Lancet, 361:374.

6. Selvanayagam JB, Porto I, Channon $\mathrm{K}$ et al.(2005): Troponin elevation after percutaneous coronary intervention directly represents the extent of irreversible myocardial injury: Insights from cardiovascular magnetic resonance imaging. Circulation, 111:1027.

7. Moon JC, De Arenaza DP, Elkington AG et al. (2004): The pathologic basis of Q-wave and non-Q-wave myocardial infarction: A cardiovascular magnetic resonance study. J Am Coll Cardiol ., 44:554.

8. Mahrholdt H, Wagner A, Holly TA et al.(2002): Reproducibility of chronic infarct size measurement by contrast-enhanced magnetic resonance imaging. Circulation, 106:2322.

9. Assami R, Ole J, Erling A et al.(2010): High Resolution Speckle Tracking Dobutamine Stress Echocardiography Reveals Heterogeneous Responses in Different Myocardial Layers: Implication for Viability Assessments. J Am Soc Echocardiogr., 23:439-447.
10. Jamal F, Kukulski T, Strotmann J et al. (2001):Quantification of the spectrum of changes in regional myocardial function during acute ischemia in closed chest pigs: an ultrasonic strain rate and strain study. J Am Soc Echocardiogr., 14:874-84.

11. Rademakers FE, Rogers WJ, Guier WH et al. (1994):Relation of regional cross-fiber shortening to wall thickening in the intact heart. Three-dimensional strain analysis by NMR tagging. Circulation, 89:1174-82.

12. Hong R, Ping-Yang Z, Ling-Ling F et al.(2012):Clinical Value of Two-Dimensional Speckle Tracking Combined with Adenosine Stress Echocardiography for Assessment of Myocardial Viability.Echocardiography,29:688-694.

13. Yan Z, Anna K, Cheuk-Man Y et al.(2005):Strain Rate Imaging Differentiates Transmural From Non-Transmural Myocardial Infarction A Validation Study Using DelayedEnhancement Magnetic Resonance Imaging. Journal of the American College of Cardiology, doi:10.1016/j.jacc.2005.05.054.

14. Weidemann F, Dommke C, Bijnens B et al. (2003): Defining the transmurality of a chronic myocardial infarction by ultrasonic strain-rate imaging. Implications for identifying intramural viability: an experimental study. Circulation, 107:883-8.

15. Karina W, Piotr H, Jarosław D et al. (2014): Feasibility and correlation of standard 2D speckle tracking echocardiography and automated function imaging derived parameters of left ventricular function during dobutamine stress test. Int J Cardiovasc Imaging, 30:729-737.

16. Trond V, Harald B, Eirik P et al. (2007): Early Prediction of Infarct Size by Strain Doppler Echocardiography After Coronary Reperfusion Journal of the American College of Cardiology. https://www.ncbi.nlm.nih.gov > pubmed

17. Joyce E, Hoogslag GE, Marsan NA (2015): Quantitative dobutamine stress echocardiography using speckle tracking analysis versus conventional visual analysis for detection of significant coronary artery disease after STSegment elevation myocardial infarction. https://www.ncbi.nlm.nih.gov > pubmed

18. Hensel KO, Jenke A, Leischik R (2014):SpeckleTracking and Tissue-Doppler Stress Echocardiography in Arterial Hypertension: A Sensitive Tool for Detection of Subclinical LV Impairment. BioMed Research International,2014:472562. 